\title{
Galactic bulges: overview
}

\author{
K. C. Freeman \\ Research School of Astronomy \& Astrophysics, The Australian National University, Australia
}

\begin{abstract}
This overview of galactic bulges begins with a discussion of the various kinds of bulges (classical, boxy/peanut-shaped, pseudo) and their likely formation mechanisms. Other specific topics include the Galactic bar/bulge and its chemical evolution, the bulge of M31, the relation between bulges and metal-poor halos (often lumped together as spheroids), the morphology-density relation and the formation of S0 galaxies, the color-structure bimodality, and scaling laws for bulges. Finally I will briefly discuss the current difficulty of forming bulgeless disk galaxies in $\Lambda$ CDM.
\end{abstract}

Keywords. Galaxy: bulge, Galaxy: halo, Galaxy: kinematics and dynamics, galaxies: bulges, galaxies: formation, galaxies: evolution, galaxies: Magellanic Clouds, galaxies: structure

\section{Introduction}

The central bulges of disk galaxies are heterogeneous, ranging from the large classical bulges of galaxies like the Sombrero galaxy (NGC 4594) to the much smaller boxy bulges of later-type disk galaxies like the Galaxy and NGC 4565 (see Figure 1). The origin of bulges is still not well understood. It seems likely now that there is more than one way to form a bulge. Many edge-on galaxies, like NGC 5907, appear to have negligible bulges, so it is clearly possible for even large galaxies to avoid the various bulge-forming processes.
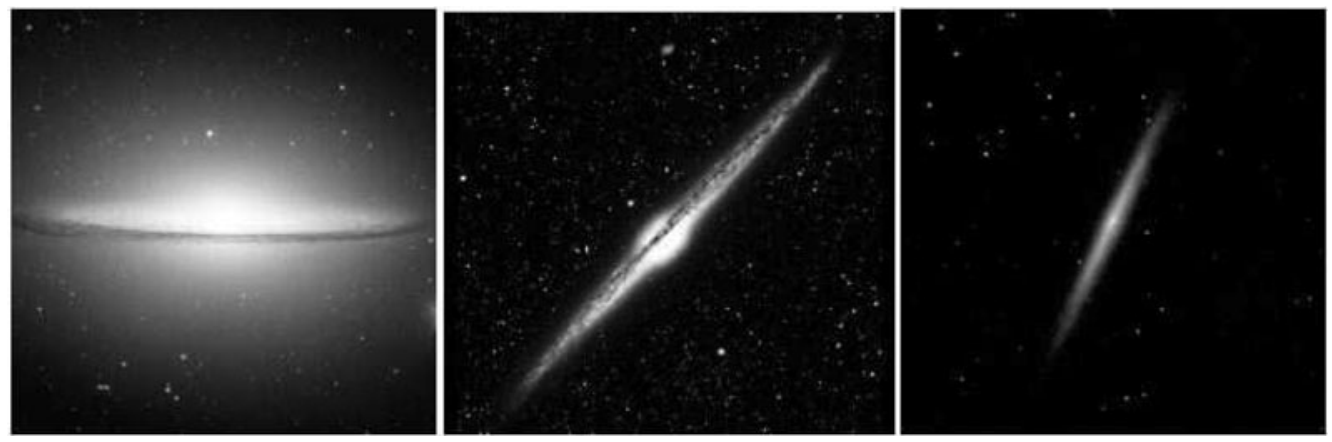

Figure 1. Three bulges. Left: NGC 4594. Center: NGC 4565. Right: NGC 5907.

The large classical bulges as in the Sombrero galaxy are believed to be generated by mergers: merger dynamics leads to bulges with the characteristic $r^{1 / 4}$ law light distribution (Sersic index 4) (e.g. Barnes 1988). These classical bulges are common in early type galaxies but become progressively rarer towards later types. They share some structural, dynamical and population properties with the lower-luminosity ellipticals

Later type galaxies like the Milky Way mostly have small boxy bulges with nearexponential surface brightness profiles rather than the $r^{1 / 4}$ profiles seen in the classical bulges (eg Courteau et al. 1996). These small bulges are probably not merger products: we know now that the boxy bulges, as in our Galaxy, are associated with bars and are 
believed to come from bar-buckling instabilities of the disk. For the theory, see Combes \& Sanders 1981 and many papers that followed. For the observational evidence for the association of boxy bulges with bars, see Kuijken \& Merrifield 1995, and Bureau \& Freeman 1999. It is theoretically possible to have systems that appear boxy or peanut-shaped when seen edge-on but are axisymmetric (e.g. Rowley 1988, Patsis et al. 2002). However, it turns out that almost all edge-on boxy/peanut bulges with observable emission lines show a characteristic figure-of-eight shape in their emission line spectra. This shape is associated with the different orbit families in the potential of a rotating bar (e.g. Bureau \& Freeman 1999).

In addition to the classical bulges and boxy/peanut bulges, there is a third kind of bulge-like structure which provides an enhancement of the surface brightness profile above the exponential disk but appears to be disk-like from its shape and kinematics. Galaxies with such enhancements may well not show any indication of having bulges when viewed edge-on. These kind of enhancements are the pseudobulges (Kormendy 1993) which are believed to be generated by secular processes associated with angular momentum transport by bars or weakly oval disks. (Recall that bars are very common. About $60 \%$ of disk galaxies show some kind of bar structure in their near-IR images: e.g. Marinova \& Jogee 2007). Several examples of pseudobulges lie well above the oblate isotropic rotator curve in the $V / \sigma-\epsilon$ plane (see Kormendy \& Kennicutt 2004). They often show active star formation within the pseudobulge region (Carollo et al. 1998).

Noordemeer \& van der Hulst (2007) derived the shape of the bulges of early-type disk galaxies from surface photometry. They concluded that these bulges have a wide range of instrinsic flattening: none is spherical, and some are close to disklike, with intrinsic axial ratios as low as 0.3 .

Turning now to the internal kinematics of bulges, there is a marked contrast between the internal motions in classical and boxy/peanut bulges. Falcón-Barroso et al. (2004) compared the classical bulge of NGC 5866 and the boxy bulge of NGC 7332. The boxy bulge shows the characteristic cylindrical rotation (i.e. the mean stellar rotation speed is almost constant with height above the equatorial plane), while the classical bulge shows a rapid decrease in rotational speed with height. This emphasises the very different stellar dynamics of these two kinds of bulges.

Bars within bars are common. About $25 \%$ of barred galaxies (including our Galaxy) have secondary bars. They are randomly orientated with respect to the major bar, so appear to be kinematically decoupled. Debattista \& Shen (2007) show how secondary bars can arise from pseudobulges; the secondard bars are potentially important for transporting matter into center for black hole fuelling or growth.

\section{The Galactic Bar/Bulge}

Our Galaxy has a small exponential boxy bulge, typical of the later-type galaxies. The stars of the bulge appear to be old ( $>10 \mathrm{Gyr}$ ) with no sign of a younger population except near the center. The metallicity distribution at $b=-6^{\circ}$ extends over the interval $[\mathrm{Fe} / \mathrm{H}]=-1.8$ to 0.2 : i.e. not very metal-rich (Zoccali et al. 2003). The bulge shows a clear metallicity gradient along its minor axis (Minniti et al. 1995). Within about 100 pc of the galactic center is a younger stellar population, with a nuclear disk $(M \sim 1.5 \times$ $\left.10^{9} \mathrm{M}_{\odot}\right)$ and a central nuclear cluster $\left(M \sim 2 \times 10^{7} \mathrm{M}_{\odot}\right)$ in the innermost 30 pc (Launhardt et al. 2002).

The Galactic bar/bulge is about $3.5 \mathrm{kpc}$ long, with an axial ratio of about 1:0.35:0.25, and points at about $25^{\circ}$ from the sun-center line into the first galactic quadrant (e.g. Rattenbury et al. 2007). Its boxy shape is not an artifact of galactic absorption: it is 
also seen clearly in inversions of the 2MASS star counts (López-Corredoira et al. 2005). López-Corredoira et al. (2007) find evidence for a longer flat bar lying in the disk of the Galaxy (dimensions about $7.8 \times 1.2 \times 0.2 \mathrm{kpc}$ ) from 2 MASS counts and red-clump stars. The central boxy bar/bulge appears to be the inner vertically extended part of this longer flatter bar. The red clump star distribution also provides evidence of an inner secondary bar with a semi-length of about 600 pc (Nishiyama et al. 2006). The distribution of stars in the inner Galaxy (boxy bulge plus longer flatter bar) may be somewhat like the distribution shown in Figure 2 for M83.
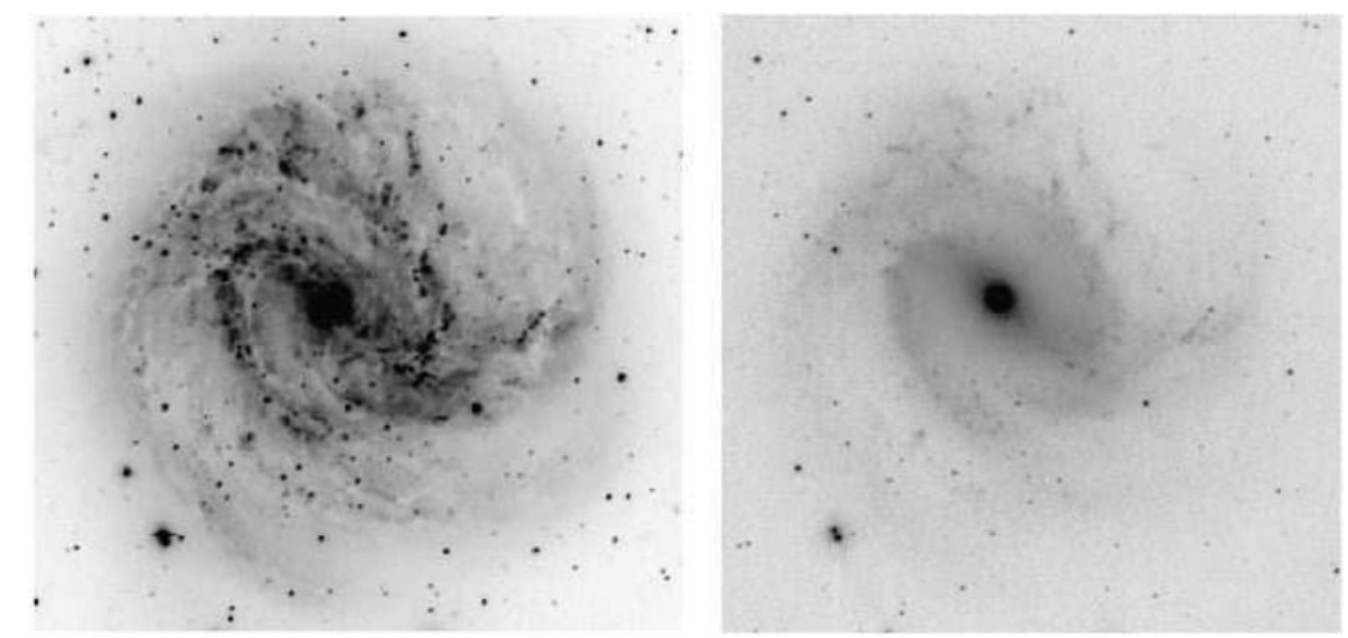

Figure 2. M83 in B (left) and K (right), showing the central bulge and the more extended bar.

How can we test whether the Galactic bulge formed through the bar buckling instability, as predicted by simulations? Do the simulations match the exponential structure and the expected cylindrical rotation? Lia Athanassoula kindly provided a simulation for analysis. Its structure and kinematics agree very well with the available surface photometry from the COBE/DIRBE near-IR observations and with the rotation and velocity dispersion of the bulge as measured from planetary nebulae (Beaulieu et al. 2000). More data on the kinematics of the Galactic bulge is needed to make a more complete comparison.

\section{Chemical Evolution of the Galactic Bulge}

The stars of the bulge are old and enhanced in $\alpha$-elements, indicating a rapid star formation history (Zoccali et al. 2006, Fulbright et al. 2007, Cunha \& Smith 2006, Lecureur et al. 2007). Chemical evolution models with supernova winds are successful in reproducing the abundance distribution and the high $[\alpha / \mathrm{Fe}]$ ratios. They require a short $(\sim 0.1$ Gyr) burst of intense star formation (Ballero et al. 2007).

We note that in the bar-buckling instability scenario for generating boxy bulges like the bulge of our Galaxy, the bulge structure may be younger that its stars, which would originally have been part of the inner disk.

Similar $\alpha$-enhancement is seen in other bulges, and is greater in the brighter bulges with larger velocity dispersions (e.g. Trager 2006). The rapid star formation history and chemical evolution of larger bulges appears to be common. 


\section{The Bulge of M31}

From its kinematics, the bulge of M31 is larger than for the Galaxy: its velocity dispersion is about $140 \mathrm{~km} \mathrm{~s}^{-1}$, compared with about $100 \mathrm{~km} \mathrm{~s}^{-1}$ for the Galactic bulge. For M31, detailed comparison of the isophotal structure of its bulge with N-body models indicates that both a classical bulge and an inner boxy bar/bulge are present (Athanassoula \& Beaton 2006, Beaton et al. 2007). As in our Galaxy, the boxy bar/bulge appears to be the extended inner part of a flat bar which extends about 1.4 times further in radius than the boxy bulge itself: see Figure 3. The metallicity distribution function of the inner bulge of M31 is very similar in shape to that of the MDF for the Galactic bulge; the M31 bulge is about 0.1 dex more metal-rich: see Figure 4.
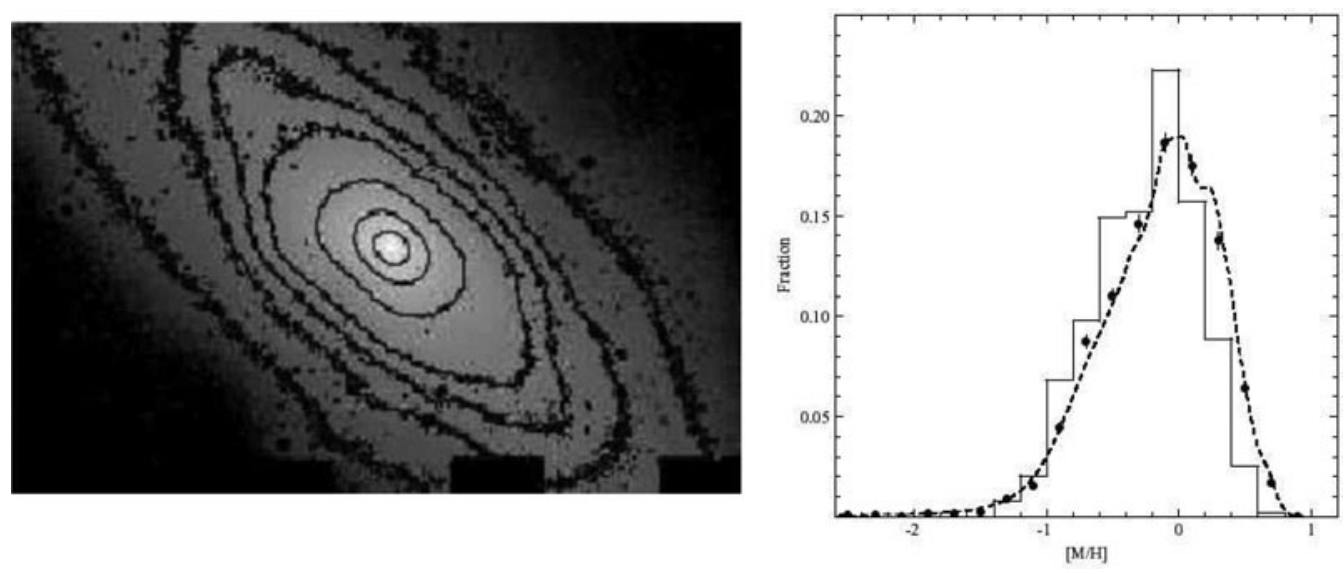

Figure 3. (left) 2MASS J-band isophotes of the inner regions of M31, showing the boxy bulge and the more extended bar (Athanassoula \& Beaton 2006, Beaton et al. 2007). Figure 4. (right) Metallicity distribution functions for the Galactic bulge (solid histogram) and the bulge of M31 (dotted curve) (Sarajedini \& Jablonka 2005).

\section{Metal-poor Halos and Bulges}

Bulges and stellar halos are often lumped together as "spheroids", but are they related cosmogonically? The Milky Way has a bulge and a metal-poor stellar halo, and so does M31. However, M33 and the LMC have negligible bulges but also have stellar halos.

Although the globular clusters in the LMC do not appear to be part of a halo component, the LMC does have a kinematically detected stellar halo. The RR Lyrae stars in the LMC (Borissova et al. 2004) have a velocity dispersion of $53 \mathrm{~km} \mathrm{~s}^{-1}$. This is the expected dispersion, given the observed rotational velocity of the disk of the LMC, and is much larger than the dispersion of the disk stars. The mean abundance of these halo RR Lyr stars is $[\mathrm{Fe} / \mathrm{H}]=-1.45$. In M33, the RR Lyrae stars and red giants again show the presence of a metal-poor halo (Sarajedini et al. 2006, McConnachie et al. 2006).

We should probably infer that the metal-poor stellar halos are not associated with bulges. It seems likely that the halos form from the accretion of many low-mass metalpoor fragile satellites, while bulge formation proceeds in several ways as discussed already.

\section{The Morphology-density Relation}

We now briefly discuss the formation of S0 galaxies. These galaxies are believed to form through the quenching of star formation in spirals (e.g. Barr et al. 2007) but the bulges 
of S0s are larger and provide a larger fraction of their light in the mean than the bulges of spirals. For example, Simien \& de Vaucouleurs (1986) showed how the bulge fraction depends on morphological type. The bulge fraction drops rapidly from S0 towards the later types, and becomes almost negligible at type Sd. S0 formation appears to involve the formation or enlargement of bulges, in addition to the quenching of star formation (see also Faber et al. 2007) so this question is relevant to our conference.

The morphology-density relation (MDR) was demonstrated by Dressler (1980) in a sample of galaxies in 55 clusters. As the mean density of the environment increases, the fraction of S0 galaxies increases and the fraction of (spirals + irregulars) decreases. He pointed out that the bulge/disk ratios, the bulge size and the bulge luminosity are larger for S0 galaxies than for spirals. For our purpose here, we note that bulge formation is potentially affected by environmental density via fast encounters, mergers, accretion and cluster tides.

Smith et al. (2005) and Postman et al. (2005) studied the evolution of the MDR out to a redshift $z \sim 1$. Like Dressler et al. (1997), they found that the evolution of the MDR is driven by a deficit of S0 galaxies and an excess of spirals and irregulars at $z \sim 1$ relative to the local population. The fraction of ellipticals shows no significant evolution from $z=1$ to $z=0$, and the evolution of the MDR is mainly via the conversion of spirals to S0s. The conversion process (and the associated increase in the luminosity and size of the bulge) are not yet well understood.

\section{The Color-structure Bimodality}

The color bimodality of galaxies is primarily an early-type/ late-type effect of old inert populations versus systems with more recent star formation (e.g. Driver et al. 2006, 2007). Disk galaxies appear in both modes: is the bimodality for these disk galaxies driven by the bulge/total luminosity fraction or by bulge type (classical or pseudo). Cameron (poster at this meeting) shows that the light distributions of the blue mode galaxies are fit by mainly low-n disklike Sersic distributions, while the red mode galaxies have typically larger Sersic indices. Drory \& Fisher (2007) argue that bulge type is important here: the blue mode galaxies are mostly pseudo-bulge systems with low bulge/total ratios, while the red mode galaxies have primarily classical bulges covering the entire range of bulge/total ratios.

\section{Scaling Laws for Bulges}

Bulges follow many scaling laws which we need to understand. The familiar fundamental plane relation provides a scaling between effective radius, surface brightness and velocity dispersion. Here are some other examples, some of which relate the properties of the bulge to the properties of the parent disk:

- the scale length of the parent disk correlates with the effective radius of the bulge (e.g. Courteau et al. 1996, Aguerri et al. 2005, Carollo et al. 2007).

- the bulge luminosity scales with the total luminosity of the parent (Carollo et al. 2007; see Figure 5).

These scaling relations suggest that the bulges of late-type galaxies come from internal evolution of their disks.

- The mass of the bulges of late-type galaxies correlates with their luminosity weighted age

- The velocity dispersion of the bulge scales with the maximum circular velocity, although the scaling may not be a simple linear law (e.g. Pizzella et al. 2005, Ho 2007, 
Courteau at this meeting). See Figure 6 . This scaling law relates the dynamics of the bulge and dark halo and may be relevant to the relation between black hole mass and velocity dispersion.

Mass-to-light ratios derived from SAURON observations (Cappellari et al. 2006) scale with the mass and velocity dispersion of the bulges. The $M / L-M$ relation is affected by variations with $M$ of the stellar age distribution, metallicity, and dark matter fraction within an effective radius. The changes in $M / L$ with mass are sufficient to explain the observed tilt of the fundamental plane. The observed evolution with redshift of the fundamental plane indicates that $M / L$ evolves with redshift, consistent with the evolution of a composite stellar population (Treu et al. 2005). The problem of the tilt of the fundamental plane appears to be reaching maturity.
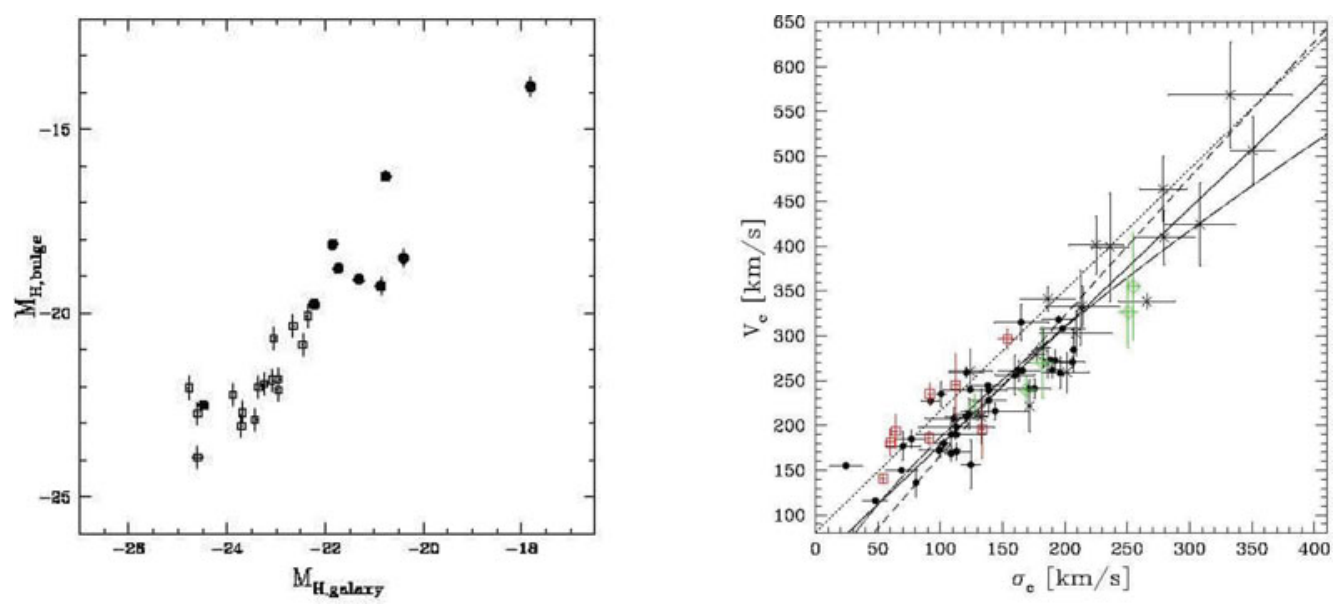

Figure 5. (left) H-band absolute magnitude of bulges vs absolute magnitude of galaxy (Carollo et al. 2007). Figure 6. (right) Velocity dispersion of bulges $v s$ circular velocity of galaxy. Filled symbols are high surface brightness galaxies, open squares low surface brightness galaxies, open diamonds and crosses ellipticals (Pizzella et al. 2005).

\section{9. $\Lambda \mathrm{CDM}$ and the Formation of Pure Disk Galaxies}

$\Lambda \mathrm{CDM}$ currently has some well-known problems on galactic scales: the satellite problem, the angular momentum problem and the central cusp problem. In the context of bulge formation, $\Lambda$ CDM produces stellar systems that are too centrally concentrated, lack angular momentum and have overly-dominant bulges. This makes it difficult to evaluate the formation of bulges through mergers in the $\Lambda$ CDM framework.

These problems may well go away when the physics of galaxy formation is better understood. But it is difficult at present for $\Lambda \mathrm{CDM}$ to generate galaxies with small or no bulges, which puts some doubt on our understanding of any kind of bulge formation in the framework of $\Lambda \mathrm{CDM}$. The problem becomes worse if many apparent bulges are pseudobulges, formed from evolution of galaxies with small or negligible classical bulges. We now briefly discuss some recent examples of simulations that are relevant to this problem.

The Abadi et al. (2003) simulation of a disk galaxy promoted the idea that a significant fraction of the oldest disk stars did not form in the disk but rather within satellites that were accreted after their orbits were dragged into the equatorial plane by dynamical friction. This simulation generated a rapidly rotating disk and an old slowly rotating 
spheroid which contributes about $60 \%$ of the light at $z=0$. About $55 \%$ of the spheroid stars is built up from accreted satellites

Springel \& Hernquist (2005) simulated the merger of two equal disk galaxies, initially composed of dark matter and gas only. Two major starbursts took place during the merger. The galaxy that is formed in this merger has a large classical bulge $(68 \%$ of the stellar mass), and an exponential disk of stars and gas.

Efforts to resolve these problems have mostly invoked feedback from star formation, but still produce few small-bulge systems. Robertson et al. (2006) were able to form near-pure disks from gas-rich mergers (gas fraction $>80 \%$ ). They use feedback processes (pressurization by the multiphase ISM) to inhibit star formation.

\section{References}

Abadi, M., Navarro, J. F., Steinmetz, M. \& Eke, V. R. 2003, ApJ 597, 21

Aguerri, J., Elias-Rosa, N., Corsini, E. M. \& Muoz-Tun, C. 2005, A $ॄ A$ 434, 109

Athanassoula, E. \& Beaton, R. 2006, MNRAS 370, 1499

Barnes, J. 1988, ApJ 331, 699

Barr, J. et al. 2007, A\&̈A 470, 173

Ballero, S. K., Matteucci, F., Origlia, L. \& Rich, R. M. 2007, A $₫ A$ 467, 123

Beaton, R., Majewski, S. R., Guhathakurta, P. et al. 2007, ApJ 658, L91

Beaulieu, S., Freeman, K., Kalnajs, A.J. et al. 2000, AJ 120, 855

Borissova, J., Minniti, D., Rejkuba, M. et al. 2004, A\&A 423, 97

Bureau, M. \& Freeman, K. 1999, AJ 118, 126

Cappellari, M., Bacon, R., Bureau, M. et al. 2006, MNRAS 366, 1126

Carollo, C. M., Stiavelli, M. \& Mack, J. 1998, AJ 116, 68

Carollo, C. M., Scarlata, C., Stiavelli, M. et al. 2007, ApJ 658, 960

Combes, F. \& Sanders, R. 1981, A\&A 96, 164

Courteau, S., de Jong, R. S. \& Broeils, A. H. 1996, ApJ 457, L73

Cunha, K. \& Smith, V. 2006, ApJ 651, 491

Debattista, V. \& Shen, J. 2007, ApJ 654, L127

Dressler, A. 1980, ApJ 236, 351

Dressler, A., Oemler, A., Couch, W.J. et al. 1997, ApJ 490, 577

Driver, S., Allen, P. D., Graham, A.W. et al. 2006, MNRAS 368, 414

Driver, S., Allen, P. D., Liske, J. \& Graham, A. W. 2007, ApJ 657, L85

Drory, N. \& Fisher, D. 2007, ApJ 664, 640

Faber, S., Willmer, C. N. A., Wolf, C. et al. 2007, ApJ 665, 265

Falcón-Barroso, J. et al. 2004, AN 325, 92

Fulbright, J., McWilliam, A. \& Rich, R. M. 2007, ApJ 661, 1152

Ho, L. 2007, ApJ 668, 94

Kormendy, J. 1993, in "IAU Symposium 153: Galactic Bulges", ed. H. DeJonghe

\& H. Habing, (Kluwer: Dordrecht), p 209

Kormendy, J. \& Kennicutt, R. 2004, ARAA 42, 603

Kuijken, K. \& Merrifield, M. 1995, ApJ 443, L13

Launhardt, R., Zylka, R. \& Mezger, P. G. 2002, A\&A 384, 112

Lecureur, A., Hill, V., Zoccali, M. et al. 2007, A\&A 465, 799

López-Corredoira, M., Cabrera-Lavers, A. \& Gerhard, O. E. 2005, A\&A A 439, 167

López-Corredoira, M., Cabrera-Lavers, A., Mahoney, T. J. et al. 2007, AJ 133, 154

McConnachie, A. et al. 2006, ApJ 647, L25

Minniti, D. 1995, MNRAS 277, 1293

Nordermeer, E. \& van der Hulst, J. 2007, MNRAS 376, 1480 
Marinova, I. \& Jogee, S. 2007, ApJ 659, 1176

Patsis, P. A., Athanassoula, E., Grosbøl, P., Skokos, Ch. 2002, MNRAS 335, 1049

Pizzella, A., Corsini, E. M., Dalla Bontà, E. et al. 2005, ApJ 631, 785

Postman, M., Franx, M., Cross, N. J. G. et al. 2005, ApJ 623, 721

Rattenbury, N., Mao, S., Sumi, T. \& Smith, M. C. 2007, MNRAS 378, 1064

Robertson, B., Bullock, J. S., Cox, T. J. et al. 2006, ApJ 645, 986

Rowley, G. 1988, ApJ 331, 124

Sarajedini, A. \& Jablonka, P. 2005, AJ 130, 1627

Sarajedini, A., Barker, M. K., Geisler, D. et al. 2006, AJ 132, 1361

Simien, F. \& de Vaucouleurs, G. 1986, ApJ 302, 564

Smith, G., Treu, T., Ellis, R. S. et al. 2005, ApJ 620, 78

Springel, V. \& Hernquist, L. 2005, ApJ 622, L9

Trager, S. 2006, astro-ph/0611378

Treu, T., Ellis, R. S., Liao, T. X. et al. 2005, ApJ 633, 174

Zoccali, M., Renzini, A., Ortolani, S. et al. 2003, A\&A 399, 931

Zoccali, M., Lecureur, A., Barbuy, B. et al. 2006, A\&A 457, L1 